\title{
Assessment methods for unsteady flow distortion in aero-engine intakes
}

\author{
Paper submitted to Aerospace Science and Technology \\ Daniel Gil-Prieto a, ${ }^{\text {a, }}$, David G. MacManus ${ }^{\mathrm{a}}$, Pavlos K. Zachos ${ }^{\mathrm{a}}$, and Abian Bautista ${ }^{\mathrm{a}}$ \\ ${ }^{a}$ Cranfield University, Cranfield, England, MK43 OAL, United Kingdom
}

\begin{abstract}
Peak events of unsteady total pressure and swirl distortion generated within S-duct intakes can affect the engine stability, even when within acceptable mean distortion levels. Even though the distortion descriptors have been evaluated in S-duct intakes, the associated flow field pattern has not been reported in detail. This is of importance since engine tolerance to distortion is usually tested with representative patterns from intake tests replicated with steady distortion generators. Despite its importance in intake/engine compatibility assessments, the spectral characteristics of the distortion descriptors and the relationship between peak unsteady swirl and both radial and circumferential total pressure distortion has not been assessed previously. The peak distortion data is typically lowpass filtered at a frequency associated with the minimum response time of the engine. However the engine design is not always known a priori in intakes investigations and a standard approach to reporting peak distortion data is needed. In addition, expensive and time-consuming tests are usually required to capture representative extreme distortion levels. This work presents a range of analyses based on Delayed Detached-Eddy Simulation and Stereo Particle Image Velocimetry data to assess these aspects of the unsteady flow distortion. The distorted pattern associated with different swirl distortion metrics is identified based on a conditional averaging technique, which indicates that the most intense swirl events are associated with a single rotating structure. . The main frequencies of the flow distortion descriptors in a representative S-duct intake are found to lie within the range in which the engine stability may be compromised. The peak total pressure and swirl distortion events are found to be not synchronous, which highlights the need to assess both types of distortion. Peak swirl and total-pressure distortion data is reported as a function of its associated time scale in a more general way that can be used in the assessment of intake unsteady flow distortion. Extreme Value Theory has been applied to predict peak distortion values beyond those measured in the available dataset, and whose measurement would otherwise require testing times two orders of magnitude longer than those typically considered.
\end{abstract}

Keywords: Extreme value theory; unsteady distortion; PIV; DDES; S-duct

\section{Nomenclature}

$A=$ S-duct cross section area, $\mathrm{mm}^{2}$

$A R=$ Area ratio, $A_{A I P} / A_{\text {in }}$

$D \quad=$ S-duct cross section diameter, $\mathrm{mm}$

DC60 = Distortion Coefficient

$f=$ Frequency, $\mathrm{Hz}$

$H \quad$ = S-duct centerline offset, $\mathrm{mm}$

$L \quad=$ S-duct axial length, $\mathrm{mm}$

$L_{s} \quad=$ S-duct length measured along the centerline, $\mathrm{mm}$

$M \quad=$ Mach number

$m \quad=$ Reciprocal of the probability, $1 / p$

$p \quad=$ Probability, $\%$

$p_{0} \quad=$ Total pressure, $\mathrm{Pa}$

$P D F^{*} \quad=$ Normalized Probability Density Function

$q \quad=$ Compressible dynamic head, $\mathrm{Pa}$

$r \quad=$ Radial coordinate from the AIP center, $\mathrm{mm}$

$R=$ S-duct cross section radius, $\mathrm{mm}$

$R_{c} \quad=$ Curvature radius of the $\mathrm{S}$-duct bend, $\mathrm{mm}$

$R D I=$ Radial Distortion Index

* Corresponding author. PhD student, Propulsion Engineering Centre, Building 52, Cranfield University, MK43 0AL, United Kingdom. E-mail: d.gilprieto@ cranfield.ac.uk 

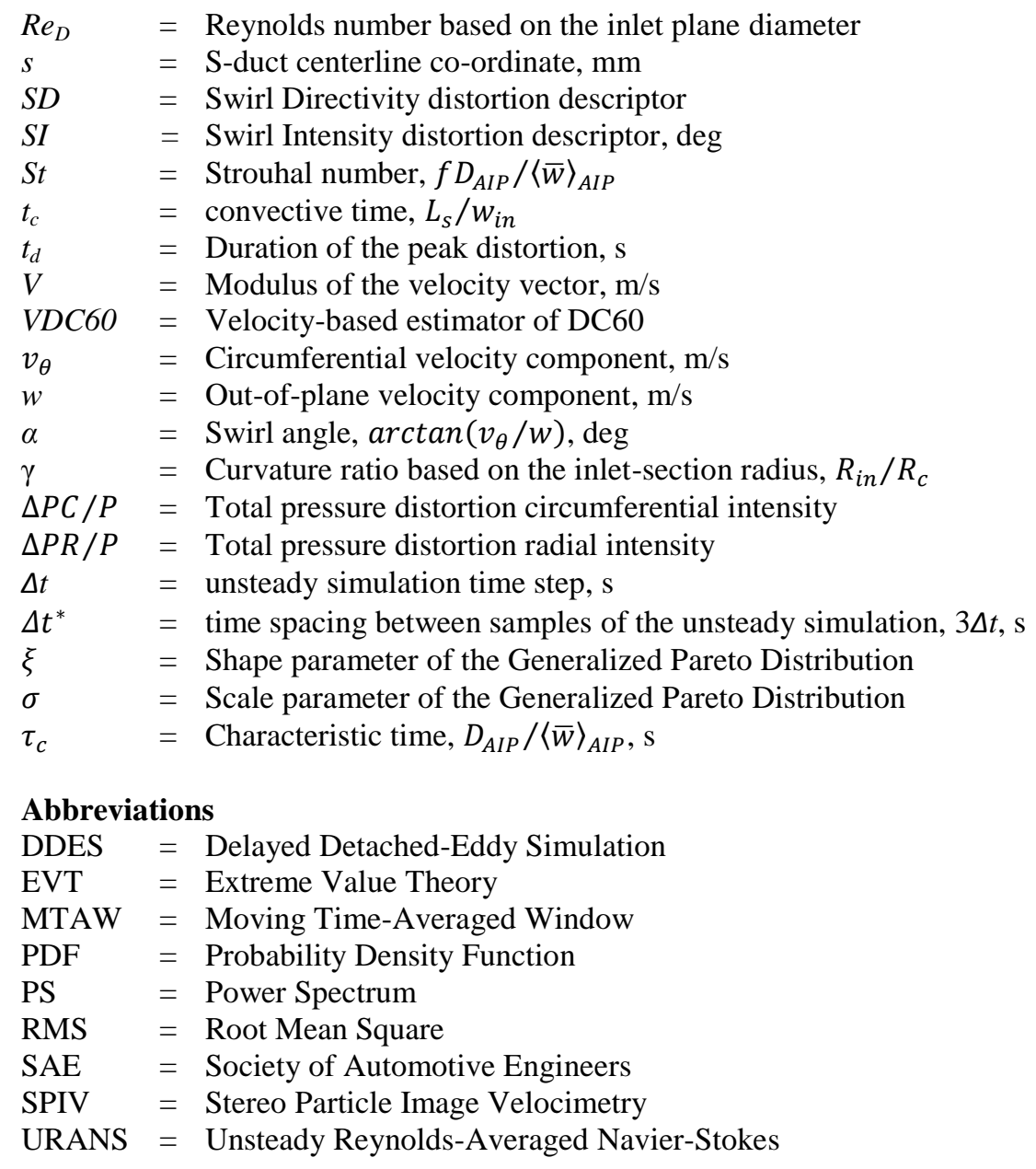

\section{Subscripts}
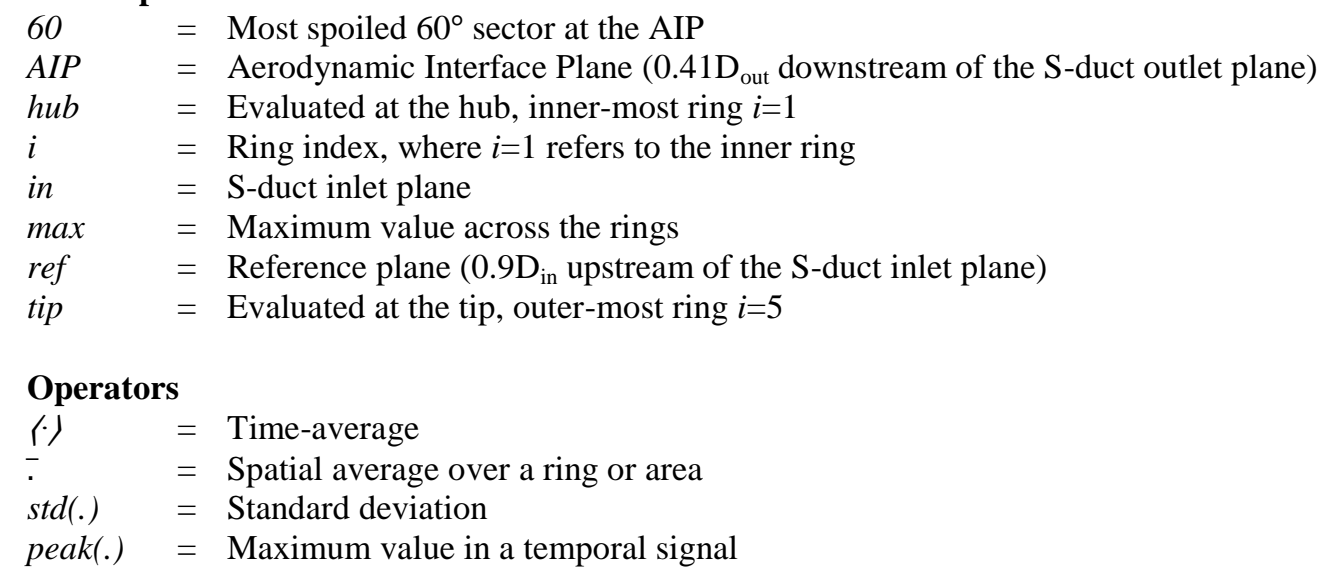

\section{Introduction}

Convoluted aero-engine intakes are needed in highly integrated power plants in which the engine is fully or partially embedded into the airframe, and are expected to play a major role in the next generation of aircraft [1,2]. A notable drawback of these configurations is the high levels of unsteady flow distortion that are delivered to the fan, as a consequence of flow separations and secondary flows within the intakes. The distortion in the flow typically reduces the surge margin and can eventually result in surge or rotating stall engine instabilities [3]. Therefore, intake/engine compatibility assessments must be addressed early in the aircraft design program to avoid extensive and expensive re-designs at later stages of the development [4]. Substantial research has been dedicated to reduce the flow distortion within these convoluted intakes with passive [5] and active [6,7] flow control, as well as through optimisation of S-duct geometries [8].

Historically, flow distortion assessments were limited to steady total pressure distortion measured with low-bandwidth pressure probes, and its effect on the fan stability is relatively well stablished [9]. However, swirl distortion more recently proved to be also a 
potential source of engine instabilities and caused time-consuming and expensive modifications in later stages of the development of several aircraft [10]. The effect of the unsteady component of the flow distortion was also identified as a major source of engine instabilities, and peak instantaneous values of the distortion descriptors that exceed the engine tolerance were reported to cause engine surge events even within acceptable mean distortion levels [11]. Consequently the peak value of the instantaneous distortion metrics has become an important parameter in inlet/engine compatibility assessments [12]. However, the fan response not only depends on the instantaneous distortion level but also on the time duration and frequency associated with the perturbation [13]. This is due to the finite response time of the fan, that needs time to adapt to the unsteady inlet conditions [14]. For perturbations with time-periods less than the fan critical response time the engine is unable to follow the inlet variations and is broadly unaffected by the unsteady component of these perturbations. In these cases, the fan is mainly sensitive to the time-averaged level of these perturbations [14]. Cousins [15] defined the critical response time for a fan rotor blade as the time for a flow particle to travel from the leading edge to the throat of the blade. Therefore the distortion data is typically low-pass filtered at the frequency associated with the minimum critical response time of the compression system [11,16-18]. However this is not always possible during the early experiments of the intake as the engine design may not be known yet. This is the case in investigations focused on the intake flow field where an engine application is not considered, and often peak distortion data is reported without any explicit consideration of the compression response time $[19,20]$.

The distorted flow within complex intakes has been widely investigated. Wellborn et al. [21] investigated the distorted flow within an S-duct intake $\left(\mathrm{H} / \mathrm{L}=0.27, \mathrm{AR}=1.52, \mathrm{~L} / \mathrm{D}_{\text {in }}=5.0\right.$, Fig. 1) with low-bandwidth instrumentation. The mean flow at the Aerodynamic Interface Plane (AIP) was characterised by a main loss region (Fig. 2a,d) and a symmetric pair of vortices (Fig. 2b,e), due to the presence of flow separation and secondary flows within the intake. Garnier [19] measured the unsteady total pressure field at the AIP of a more aggressive $S$-duct intake $\left(\mathrm{H} / \mathrm{L}=0.49, \mathrm{AR}=1.52, \mathrm{~L} / \mathrm{D}_{\mathrm{in}}=4.95\right)$ using 40 high-bandwidth pressure transducers. At $\mathrm{M}_{\mathrm{AIP}}=0.2$ $\left(\operatorname{Re}_{\mathrm{D}}=7.5 \times 10^{5}\right)$ the spectral analysis revealed a dominant unsteady structure that consisted of a lateral movement of the main loss region (Fig. 2a,d) associated with a frequency of $\mathrm{St}=0.48$. A region of high unsteadiness at the upper boundary of the mean loss region was also reported and associated with frequencies between $\mathrm{St}=0.60-1.09$ [19]. MacManus et al. [20] simulated the flow within the same non-dimensional geometries as Wellborn et al. [21] $(\mathrm{H} / \mathrm{L}=0.27)$ and Garnier [19] $(\mathrm{H} / \mathrm{L}=0.49)$ using Delayed Detached-Eddy Simulation (DDES) methods at two flow conditions of $\mathrm{M}_{\mathrm{AIP}}=0.36\left(\operatorname{Re}_{\mathrm{D}}=1.8 \times 10^{6}\right)$ and $0.18\left(\operatorname{Re}_{\mathrm{D}}=1.1 \times 10^{6}\right)$. The centreline offset $\mathrm{H} / \mathrm{L}$ had a significant effect on the peak levels of radial distortion RDI, which in the high offset duct were twice those in the low offset configuration, while the peak levels of circumferential distortion $\overline{\triangle P C / P}$ were relatively insensitive to $\mathrm{H} / \mathrm{L}$. The Mach number $\mathrm{M}_{\mathrm{AIP}}$ had a major effect in the value of these descriptors, and at $\mathrm{M}_{\mathrm{AIP}}=0.36$ both RDI and $\overline{\Delta P C / P}$ were about four times greater compared with the $\mathrm{M}_{\mathrm{AIP}}=0.18$ case [20]. The work by Zachos et al. [22] provided a breakthrough in the experimental methods used to measure flow distortion within S-duct intakes. For the first time SPIV was applied to quantify the unsteady velocity vector field at the AIP, with approximately 250 times more data points compared with conventional techniques based on 40 pressure transducers. Two Sducts were considered with the same non-dimensional geometry as Wellborn et al. [21] and Garnier [19], and the inlet Mach numbers ranged from $0.27\left(\operatorname{Re}_{\mathrm{D}}=0.6 \times 10^{6}\right)$ to $0.60\left(\mathrm{Re}_{\mathrm{D}}=1.4 \times 10^{6}\right)$. This work focused on the analysis of the unsteady swirl distortion and the peak values of $\overline{S I}$ were $15.6^{\circ}$ and $10.7^{\circ}$ for the high and low offset ducts at $\mathrm{M}_{\mathrm{ref}}=0.60$, respectively. The effect of the Mach number on the swirl distortion descriptors was minor. Gil-Prieto et al. [23] demonstrated the need for unsteady measurements for swirl distortion assessments, since the peak SI values were one order of magnitude greater than the static values evaluated from the mean flow field. These previous studies $[20,22,23]$ used the peak value as a metric to quantify the unsteady flow distortion. However, peak distortion is an inconsistent metric since the observed magnitude depends on the acquisition frequency and the size of the dataset [12]. Jacocks et al. [12] first proposed to use Extreme Value Theory (EVT) [24] to predict the maximum level of a given distortion metric to be expected after a given operating time with well-defined confidence intervals. Jacocks et al. indicated that the application of EVT to a short dataset could give similar results to the conventional approach of determining the peak value in a measured dataset 30 times larger. Therefore, EVT offers the possibility to reduce the expensive tests typically used for peak distortion detection [11].

Previous distortion studies in S-duct intakes $[19,20]$ did not take into account the duration of the peak distortion event, which is of prime importance for the response of the fan [14]. Moreover, there is a lack of knowledge about the instantaneous flow field associated with the peak distortion events. Finally, the relationship between swirl and both circumferential and radial total pressure instantaneous distortion has not been investigated. The aim of this paper is to present a range of analysis approaches pertinent to the evaluation of unsteady intake flow distortion. This includes the evaluation of the peak distortion levels for different time durations, the relationship between unsteady swirl and total pressure distortion, the use of EVT to estimate peak distortion levels as well as aerodynamic analysis to identify the flow field characteristics that promote the greatest swirl distortion events.

\section{Methodology}

\subsection{Studied case}

The S-duct configuration considered in this work has the same non-dimensional geometry as the intake investigated by Garnier [19].The S-duct has a circular cross-section and the main geometrical parameters are an area ratio of $A R=1.52$, a length of $L / D_{\text {in }}=4.95$ and a centreline offset of $\mathrm{H} / \mathrm{L}=0.49$ (Fig. 1). The S-duct centreline is composed of two consecutive $52^{\circ}$ arcs with curvature ratios $\left(\mathrm{R}_{\mathrm{in}} / \mathrm{R}_{\mathrm{c}}\right)$ of $\gamma=0.16$. The computational and experimental results presented in this work correspond to a reference Mach number of $\mathrm{M}_{\mathrm{ref}}=0.27$ and a $\operatorname{Re}_{\mathrm{D}}=7.1 \times 10^{5}$. 


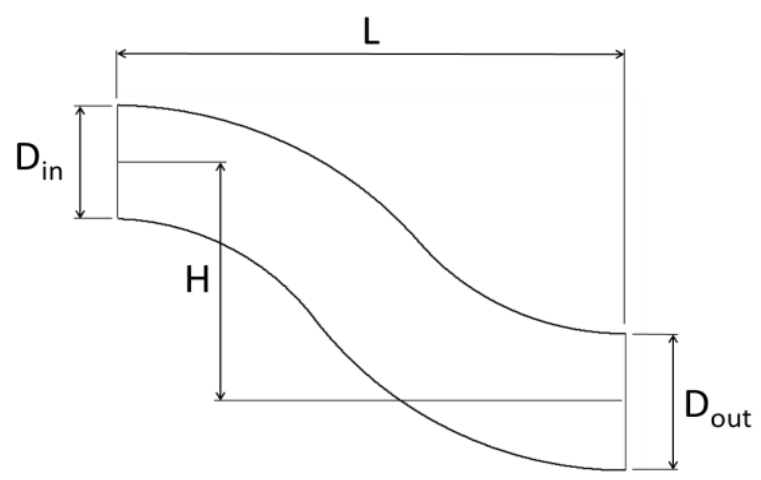

Fig. 1 S-duct geometry sketch

\subsection{Stereo Particle Image Velocimetry}

A detailed description of the experimental facility and methods is provided by Zachos et al. [22] and only key aspects are discussed here. A transparent section is attached to the outlet plane of the S-duct to permit optical access for both SPIV laser and cameras. The measurements are performed at the AIP which is located $0.41 \mathrm{D}_{\text {AIP }}$ downstream of the S-duct outlet plane. The seeding particles were illuminated with a dual cavity, frequency-doubled Nd:YAG laser. Two TSI PowerView Plus 8MP cameras were used in a stereoscopic configuration and the acquisition rate was approximately $3.5 \mathrm{~Hz}$. About 14000 velocity vectors were obtained at the AIP, which resulted in a spatial resolution of approximately $1.1 \mathrm{~mm}\left(0.007 \mathrm{D}_{\mathrm{AIP}}\right)$. A disparity correction [25] was applied to account for the potential misalignment between the laser light sheet and the calibration target. The SPIV measurements uncertainty was estimated with the procedure proposed by Raffel et al. [26] as approximately $6 \%$ and $8 \%$ for the in-plane and out-of-plane velocity components, respectively. A dataset of 1000 snapshots was considered sufficient to provide statistically converged results [22]. Only SPIV data within $95 \%$ of the radius of the AIP have been analysed to avoid the effect of spurious vectors near the walls due to laser-light reflections. The experiment is conducted with the S-duct intake in isolation without the presence of downstream rotating turbomachinery, as is the case in similar investigations [19,21]. This is the established practice in the industry for the evaluation of the intake performance during the early stages of a propulsion system development [9]. However, it is worth noting that the presence of the engine downstream of the intake is expected to have an effect on the upstream flow field. Typically the engine has a stabilising effect by delaying flow separation and reducing flow distortion generated within the intake, as reported for example by Motycka [27] and Hodder [28]. Therefore it is generally assumed that tests of isolated intakes provide a conservative measure of the distortion levels [27]. The results presented in this work hence represent the intrinsic flow instabilities generated within the S-duct in the absence of the engine turbomachinery.

\subsection{Delayed Detached-Eddy Simulation}

The details of the DDES method, verification and validation were extensively discussed by Gil-Prieto et al. [29] and only key aspects are reported here. The numerical simulation used a DDES unsteady method, which applies the Unsteady Reynolds Averaged Navier-Stokes (URANS) equations in the boundary layer and the Large Eddy Simulation method in the highly unsteady regions away from the wall [30]. The k- $\omega$ SST model was used for the URANS turbulence modelling. The momentum, density, energy and turbulence equations were spatially discretised with a third order discretisation scheme, while the pressure equations were solved with a second order scheme. The temporal formulation was based on a second order implicit scheme. The measured total pressure profile at the reference plane located $0.9 \mathrm{D}_{\text {in }}$ upstream of the S-Duct inlet plane was matched in the DDES [29]. A uniform static pressure boundary condition was applied at the outlet of the domain in order to match the measured mass flow rate. A baseline structured mesh of 5 million nodes was generated with an $\mathrm{H}$-grid structure in the centre of the S-duct section and an O-grid structure around the walls. The mesh was refined near the walls in order to ensure the $y^{+}$was smaller than 1 over the full domain, with an expansion ratio off the wall of 1.05 . The time step was set to $\Delta \mathrm{t}=1.2 \times 10^{-5} \mathrm{~s}\left(\Delta \mathrm{t} / t_{c}=3.72 \times 10^{-3}\right)$, and each time step was solved with 20 sub-iterations which resulted in maximum residuals of the order of $10^{-6}$ at the end of each time step computation [29]. A grid sensitivity analysis was performed with three meshes of $2.5,5$ and 10 million nodes at $\mathrm{M}_{\mathrm{ref}}=0.60$ and minor discrepancies were found between the areaaveraged SI (Eq. 1) statistics. For example, $\langle\overline{S I}\rangle$ was $10.2^{\circ}, 9.9^{\circ}$ and $9.3^{\circ}$ for the fine, medium and coarse meshes, respectively, and similar results were obtained for $\operatorname{std}(\overline{S I})$ and $\max (\overline{S I})[29]$. The sensitivity to the time-step $\Delta$ t was also minor, and similar levels of $\langle\overline{S I}\rangle, \operatorname{std}(\overline{S I})$ and $\max (\overline{S I})$ where obtained for the medium mesh with a doubled time-step. The first $115 \mathrm{t}_{\mathrm{c}}$ of the unsteady DDES simulation are not considered for the analysis to remove any effect of the transition between the RANS and DDES solutions. The results presented in this work are based on approximately $50 \mathrm{t}_{\mathrm{c}}$ for which the flow field is statistically converged [29].

\subsection{Distortion descriptors}

In the present work the swirl distortion is quantified with the established descriptors proposed by the Society of Automotive Engineers [10]. For the evaluation of the descriptors 5 equal-area rings have been considered at the AIP for consistency with the typical industry practice [9], and the data has been linearly interpolated at 72 azimuthal positions at each ring. The swirl angle circumferential distribution at the $i$-th ring, $\alpha_{i}(\theta)$, is characterised with the Swirl Intensity $S I_{i}$ (Eq. 1) and Swirl Directivity $S D_{i}($ Eq. 2 ) 
[10]. The SI represents the averaged absolute swirl angle, while the SD is an indicator of the overall sense of rotation of the swirling flow. For example, $\mathrm{SD}=0$ indicates a twin-swirl distribution while $\mathrm{SD}=1$ suggests the presence of a positive bulk swirl. In the present work, the swirl angle is assumed positive in the counter-clock wise direction when the AIP is considered from downstream.

$$
\begin{gathered}
S I_{i}=\frac{\int_{0}^{2 \pi}\left|\alpha_{i}(\theta)\right| d \theta}{2 \pi} \\
S D_{i}=\frac{\int_{0}^{2 \pi} \alpha_{i}(\theta) d \theta}{\int_{0}^{2 \pi}\left|\alpha_{i}(\theta)\right| d \theta}
\end{gathered}
$$

The well-known Distortion Coefficient DC60 (Eq. 3) is used to quantify the circumferential total pressure distortion [31]. A modified formulation of the DC60 has been derived assuming incompressible flow and uniform static pressure at the AIP, and this new metric is referred to as Velocity Distortion Coefficient (VDC) (Eq. 4). The VDC60 has been derived as a distortion metric for those situations when only velocity data is available as is the case in SPIV experiments, and will be further discussed in Section 3.6.2.

$$
\begin{aligned}
D C 60 & =\frac{\overline{p_{0_{A I P}}}-\overline{p_{0_{60}}}}{\overline{q_{A I P}}} \\
V D C 60 & =\frac{\overline{V^{2}{ }_{A I P}}-\overline{V^{2}}{ }_{60}}{\overline{V^{2}{ }_{A I P}}}
\end{aligned}
$$

A more sophisticated formulation to quantify total pressure distortion has been proposed by the SAE [9] to differentiate between the circumferential and radial components of the $p_{0}$-distortion at different rings. The radial $p_{0}$-distortion intensity at the $i$-th ring, $\left(\frac{A P R}{P}\right)_{i}$, represents the difference between the averaged total pressure at the $i$-th ring, $\overline{p_{0_{l}}}$, and the area-averaged AIP value $\overline{p_{0_{A I P}}}$ (Eq. 5). At each $i$-th ring there may be, in general, multiple spoiled regions where the total pressure $p_{0_{i}}(\theta)$ is lower than $\overline{p_{0_{l}}}$. The circumferential intensity at the $i$-th ring, $\left(\frac{A P C}{P}\right)_{i}$, is calculated using the averaged value $\overline{p_{0_{l, k}}}$ in the most-spoiled $k$-th region of minimum $\overline{p_{0}, k} \theta_{i, k}$ (Eq. 6), where $\theta_{i, k}$ represents the circumferential extent of the $k$-th spoiled region. To reduce the radial and circumferential intensity distributions to one single value per snapshot, the Radial Distortion Index (RDI) (Eq. 7) and maximum circumferential distortion $\left(\frac{A P C}{P}\right)_{\max }$ (Eq. 8) are used.

$$
\begin{gathered}
\left(\frac{A P R}{P}\right)_{i}=\frac{\overline{p_{0_{A I P}}}-\overline{p_{0_{l}}}}{\overline{p_{0_{A I P}}}} \\
\left(\frac{A P C}{P}\right)_{i}=\frac{\overline{p_{0_{l}}}-\overline{p_{0_{l, k}}}}{\overline{p_{0_{l}}}} \\
R D I=\left\{\begin{array}{l}
-\left(\frac{A P R}{P}\right)_{1} ; \quad \text { if }\left(\frac{A P R}{P}\right)_{1}>\left(\frac{A P R}{P}\right)_{5}, \text { hub dominated } \\
\left(\frac{A P R}{P}\right)_{5} ; \quad \text { if }\left(\frac{A P R}{P}\right)_{1}<\left(\frac{A P R}{P}\right)_{5}, \text { tip dominated } \\
\left(\frac{A P C}{P}\right)_{\max }=\max \left\{\left(\frac{A P C}{P}\right)_{i}\right\}_{i=1, \ldots, 5}
\end{array}\right.
\end{gathered}
$$

\subsection{Extreme Value Theory}

Extreme Value Theory (EVT) permits the estimation of the probability associated with the extreme events of a process $X$ based on a reduced sample of $n$ independent and identically distributed observations $\left\{X_{1}, \ldots, X_{n}\right\}$ [24]. EVT was introduced in the context of flow distortion to predict peak distortions by Jacocks et al. [12]. In the present work, the EVT is applied with a threshold model approach in which the events are considered as excesses if they exceed a certain threshold $U$ [24]. When the number of observations $n$ and the threshold $U$ are large enough, the limit distribution of the $k$ excesses $Y_{i}$ (Eq. 9) approaches a Generalized Pareto Distribution (Eq. 10), where $\xi$ and $\sigma$ are the shape and scale parameters of the model, respectively. When $\xi<0$ the distribution of excesses over the threshold has an upper bound at $U-\sigma / \xi$. The model parameters $\xi$ and $\sigma$ are estimated to maximise the probability of the observed data, through the maximisation of the log-likelihood function (Eq. 11) [24].

$$
Y_{i}=\left.\left(X_{i}-U\right)\right|_{X_{i}>U}
$$




$$
\begin{gathered}
H(y)=P\{Y \leq y\}=1-\left(1+\frac{\xi y}{\sigma}\right)^{-1 / \xi} \\
l(\xi, \sigma)=\sum_{i=1}^{k} \log \left(\frac{d H}{d y}\left(Y_{i}, \sigma, \xi\right)\right)=-k \log (\sigma)-(1+1 / \xi) \sum_{i=1}^{k} \log \left(1+\frac{\xi Y_{i}}{\sigma}\right)
\end{gathered}
$$

The extreme value $x_{m}$ that is exceeded on average once every $m$ observations is usually called $m$-observation return level, and can be estimated with the model parameters $\xi$ and $\sigma$ and the probability of exceeding the threshold $\zeta_{u}=k / n$ (Eq. 12) [24]. Variance errors for $x_{m}$ can be derived with the delta method (Eq. 13) using the gradient $\nabla x_{m}^{T}$ (Eq. 14) and the variance-covariance matrix $V C$ (Eq. 15) that gathers the variance errors of the different model parameters (Eq. 16-19) [32]. The 95\% confidence intervals $C I$ for $x_{m}$ can then be obtained assuming that $x_{m}$ follows a normal distribution and using a quantile $z_{\alpha / 2}=1.96$ (Eq. 20).

$$
\begin{aligned}
& x_{m}=U+\frac{\sigma}{\xi}\left[\left(m \zeta_{u}\right)^{\xi}-1\right] \\
& \operatorname{Var}\left(x_{m}\right)=\nabla x_{m}^{T} V C \nabla x_{m} \\
& \nabla x_{m}^{T}=\left[\frac{\partial x_{m}}{\partial \zeta_{u}}, \frac{\partial x_{m}}{\partial \sigma}, \frac{\partial x_{m}}{\partial \xi}\right] \\
& V C=\left[\begin{array}{ccc}
\operatorname{Var}\left(\zeta_{u}\right) & 0 & 0 \\
0 & \operatorname{Var}(\sigma) & \operatorname{Covar}(\xi, \sigma) \\
0 & \operatorname{Covar}(\xi, \sigma) & \operatorname{Var}(\xi)
\end{array}\right] \\
& \operatorname{Var}\left(\zeta_{u}\right)=\zeta_{u}\left(1-\zeta_{u}\right) / n \\
& \operatorname{Var}(\xi)=\frac{(1+\xi)^{2}}{k} \\
& \operatorname{Var}(\sigma)=2(1+\xi) \sigma^{2} / k \\
& \operatorname{Covar}(\xi, \sigma)=-(1+\xi) \sigma / k \\
& C I=x_{m} \pm z_{\alpha / 2} \sqrt{\operatorname{Var}\left(x_{m}\right)}
\end{aligned}
$$

\section{Results}

\subsection{Flow field at the Aerodynamic Interface Plane}

The experimental validation for the S-duct simulation considered in this study at $\mathrm{M}_{\mathrm{ref}}=0.27$ was reported in detail by Gil-Prieto et al. [29], and the time-averaged and fluctuating velocity fields at the AIP are only briefly described in this section for completeness. The time-averaged $w$-velocity field at the AIP is characterised by a main loss region (Fig. 2a,d). The in-plane mean velocity field shows the presence of the expected symmetric pair of vortices usually found in S-duct intakes due to the curvature of the geometry (Fig. 2b,e). The flow field in these kinds of intakes is highly unsteady [22] and the greatest $w$-velocity fluctuations at the AIP occur at the centre of the section, with values as high as $\operatorname{std}(w) /\langle\bar{w}\rangle_{A I P}=0.23$ (Fig. 2c). Overall, there is a good agreement between the timeaveraged and standard-deviation flow fields for DDES and SPIV (Fig. 2), as demonstrated by Gil-Prieto et al. [29]. For example, the minimum values of $\langle w\rangle /\langle\bar{w}\rangle_{A I P}$ are approximately 0.76 and 0.75 , and the maximum values of std $(w) /\langle\bar{w}\rangle_{A I P}$ are 0.23 and 0.22 , for DDES and SPIV, respectively [29].

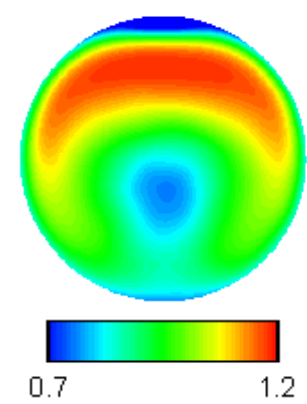

(a) $\langle w\rangle /\langle\bar{w}\rangle_{A I P}$, DDES

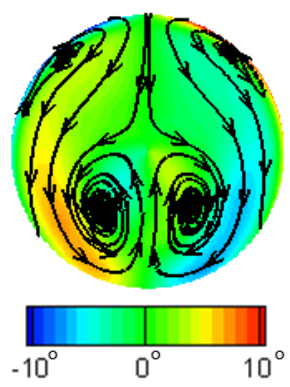

(b) $\langle\alpha\rangle$, DDES

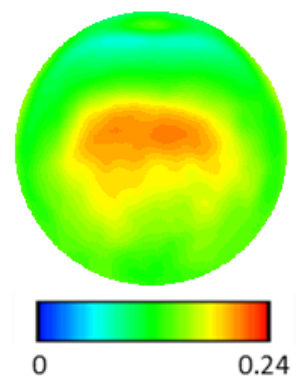

(c) $\operatorname{std}(w) /\langle\bar{w}\rangle_{A I P}$, DDES 


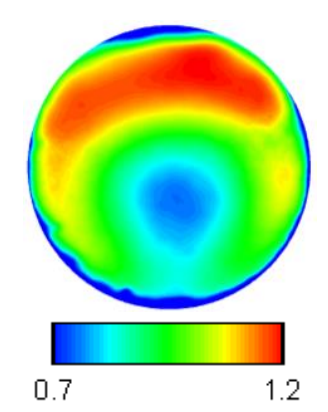

(d) $\langle w\rangle /\langle\bar{w}\rangle_{A I P}$, SPIV

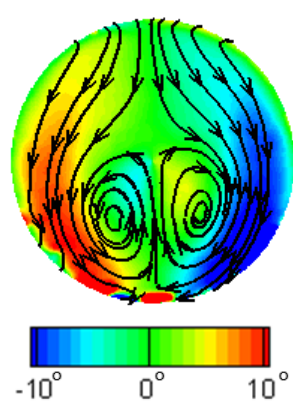

(e) $\langle\alpha\rangle$, SPIV

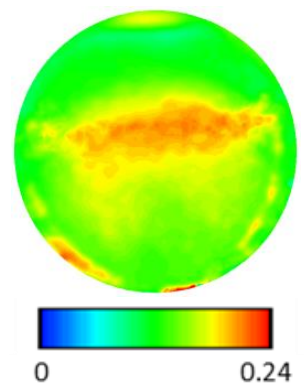

(f) $\operatorname{std}(w) /\langle\bar{w}\rangle_{A I P}$, SPIV

Fig. 2 Flow field statistics at the AIP for DDES (top) and SPIV (bottom), including the time-averaged $w$-velocity (left), timeaveraged swirl angle (centre) and standard-deviation of the $w$-velocity (right) [29]

\subsection{Flow field associated with maximum swirl distortion}

Several swirl distortion descriptors have been proposed to quantify and characterize the swirl distortion (Section 2.4), and have been recently used in flow distortion investigations in S-duct intakes [22]. Gil-Prieto et al. [23] showed that the greatest values of Swirl Intensity in these kinds of S-duct intakes occur when the flow field deviates from the mean twin-swirl distribution $\left(\mathrm{SD}_{2}=0\right)$ and moves towards bulk-swirl configurations $\left(\left|\mathrm{SD}_{2}\right|=1\right)$ in which the swirling flow in the ring features a single direction $(\mathrm{Fig}$. 3a,b). These descriptors are evaluated at discrete radial positions, and then the value is tentatively associated with the secondary flow pattern at the AIP. However, there is a lack of information about the detailed AIP flow field characteristics associated with the different values of these descriptors. The knowledge of these patterns is of importance, since engine distortion tolerance is typically assessed replicating the most critical distorted patterns with steady-state distortion generators [33]. In this section the flow field is conditionally averaged based on the different values of the Swirl Directivity descriptor. The ultimate focus of this section is to identify the most representative unsteady swirl distortion patterns generated in the considered S-duct intake. For this study the swirl descriptors are evaluated at $\mathrm{r} / \mathrm{R}_{\mathrm{AIP}}=0.55$, which corresponds to the second ring in a conventional 5 equal area rings arrangement usually considered in the industry [9]. However qualitatively similar results are found for the different rings. For the conditional averaging analysis the positive range of $\mathrm{SD}_{2}$ has been divided into three regions: $[0.00,0.33),[0.33,0.66)$, and $[0.66,1.00]$. For the DDES data, when $\mathrm{SD}_{2}$ is within $[0,0.33$ ) the conditionally averaged flow field (Fig. 4a) deviates only slightly from the mean flow symmetric distribution associated with $\mathrm{SD}_{2}=0$ (Fig. 2b). The left, counter-clockwise vortex becomes dominant as $\mathrm{SD}_{2}$ increases (Fig. $4 \mathrm{~b}$ ) until eventually a single anticlockwise rotating cell covers the AIP (Fig. 4c). Therefore, the pattern that promotes the greatest $\mathrm{SI}_{2}$ is characterised by a single swirling structure that spans over the AIP (Fig. 4c). The main loss region follows the movement of the dominant vortex which results in a non-symmetric $w$-velocity distribution (Fig. 4c). The experimental SPIV data show similar flow field characteristics as the DDES (Fig. 4d,e,f). Similar characteristics, with opposite senses of rotation, are observed in both the DDES and SPIV data when the conditional-averaging is applied for the range of negative $\mathrm{SD}_{2}[0.00,-0.33),[-0.33,-0.66)$ and $[-0.66,-1.00]$. These results further validate the unsteady data predicted by the DDES, and demonstrate the capability of these simulations as a useful tool for intake distortion assessments.

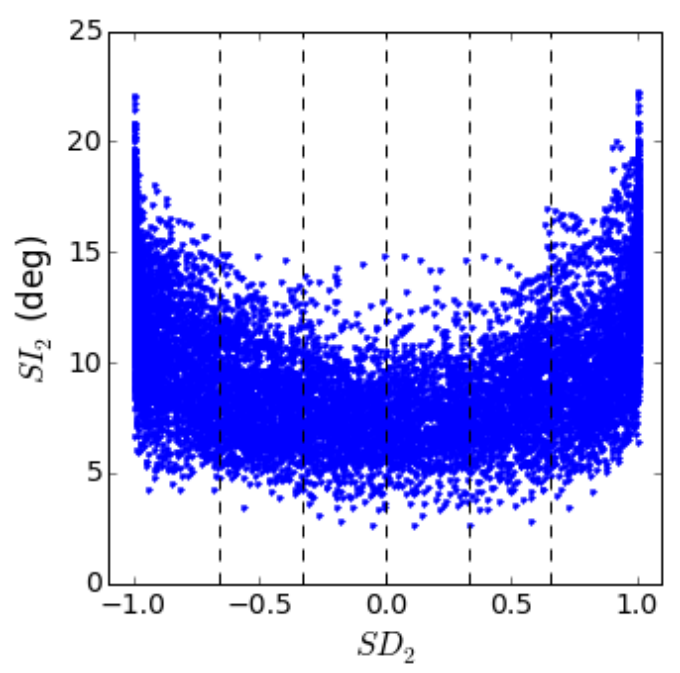

(a) $\mathrm{SI}_{2}-\mathrm{SD}_{2}$ map, DDES

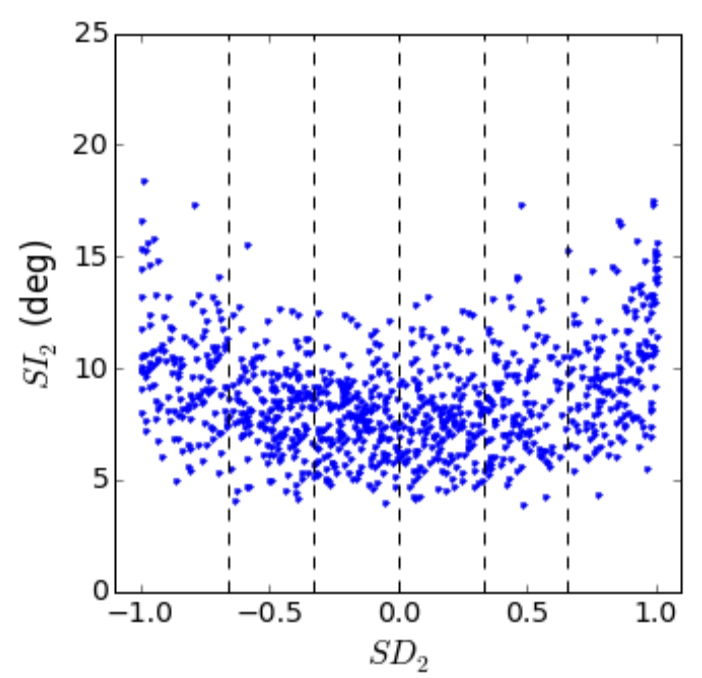

(b) $\mathrm{SI}_{2}-\mathrm{SD}_{2}$ map, SPIV

Fig. $3 \mathrm{SI}_{2}-\mathrm{SD}_{2}$ cloud map for DDES (left) and SPIV (right) 

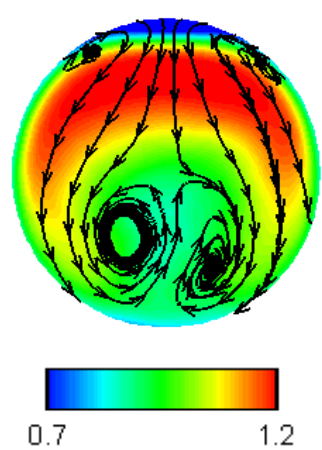

(a)

$\left.\langle\boldsymbol{w}\rangle\right|_{\mathrm{SD}_{2} \in[0,0.33),} /\langle\overline{\boldsymbol{w}}\rangle_{\boldsymbol{A I P}}, \mathrm{DDES}$
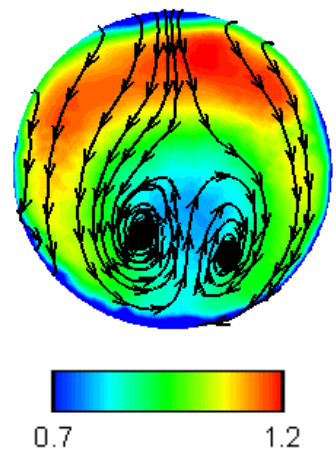

(d) $\left.\langle\boldsymbol{w}\rangle\right|_{\mathrm{SD}_{2} \in[0,0.33),} /\langle\overline{\boldsymbol{w}}\rangle_{A I P}, \mathrm{SPIV}$
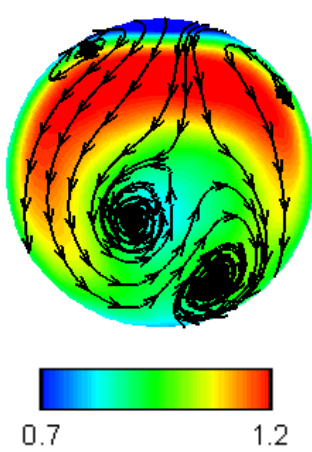

(b) $\left.\langle\boldsymbol{w}\rangle\right|_{\mathrm{SD}_{2} \in[0.33,0.66),} /\langle\overline{\boldsymbol{w}}\rangle_{A \boldsymbol{I} \boldsymbol{P}}$, DDES
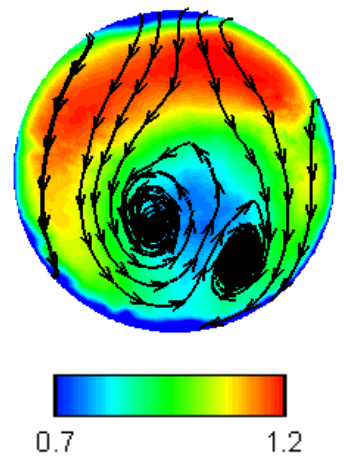

(e) $\left.\langle\boldsymbol{w}\rangle\right|_{\mathrm{SD}_{2} \in[0.33,0.66),} /\langle\overline{\boldsymbol{w}}\rangle_{A I P}$, SPIV
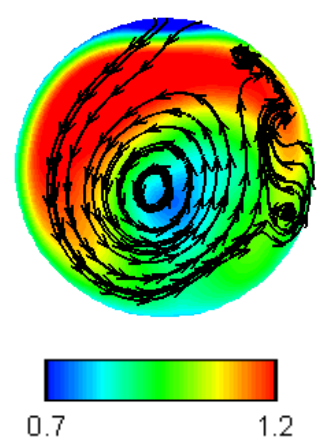

(c) $\left.\langle\boldsymbol{w}\rangle\right|_{\mathrm{SD}_{2} \in[0.66,1]}, /\langle\overline{\boldsymbol{w}}\rangle_{A I P}$, DDES
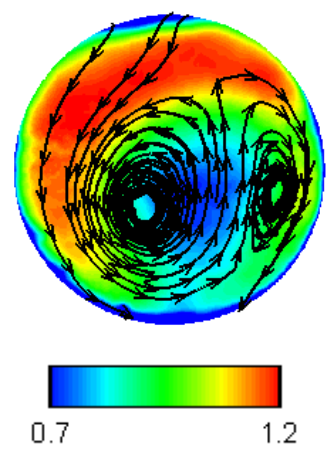

(f) $\left.\langle\boldsymbol{w}\rangle\right|_{\mathrm{SD}_{2} \in[0.66,1],} /\langle\overline{\boldsymbol{w}}\rangle_{A I P}, \mathrm{SPIV}$

Fig. 4 Conditionally-averaged $w$-velocity distribution at the AIP based on $\mathrm{SD}_{2}$

\subsection{Total pressure and swirl distortion relationship}

Total pressure and swirl distortion distortions are usually investigated separately due to the difficulties associated with their simultaneous measurement, and there is a lack of knowledge on the coupled behavior of unsteady total pressure and swirl distortion [10]. In this section the relationship between unsteady total pressure and swirl distortion is evaluated using DDES computational data through the joint-PDFs of total pressure and swirl distortion descriptors. As opposed to the conventional cloud maps [19,20], jointPDF maps differentiates between common and rare, but still potent, distortion events. In particular, the correlation between Swirl Intensity $(\mathrm{SI})$, and circumferential $\left((\Delta \mathrm{PC} / \mathrm{P})_{\max }\right)($ Fig. 5a) and radial total pressure distortion (RDI) (Fig. 5b) is assessed. RDI is defined as positive in those events in which it is evaluated at the tip $\left(r / R_{\text {AIP }}=0.94\right)$, while negative RDI values are used for those events in which the radial distortion is dominant in the hub ( $\left.\mathrm{r} / \mathrm{R}_{\mathrm{AIP}}=0.32\right)$ (Eq. 7), as proposed by Kidman et al. [34]. For the calculation of the joint-PDF the maps are divided in 60 partitions with a resolution of approximately $0.0004,0.0007$ and $0.4^{\circ}$ for $(\Delta \mathrm{PC} / \mathrm{P})_{\max }, \mathrm{RDI}$ and $\mathrm{SI}_{2}$, respectively.

The $\mathrm{SI}_{2}-(\Delta \mathrm{PC} / \mathrm{P})_{\max }$ joint-PDF map indicates that these descriptors are not significantly correlated (Fig. 5a), and peak values of $(\triangle \mathrm{PC} / \mathrm{P})_{\max }$ are not synchronous with extreme $\mathrm{SI}_{2}$ events. For example, peak $\mathrm{SI}_{2}$ values of around $22^{\circ}$ are associated with mid values of $(\Delta \mathrm{PC} / \mathrm{P})_{\max }=0.010$ (Fig. 5a). On the other hand, peak $(\Delta \mathrm{PC} / \mathrm{P})_{\max }$ values around 0.023 are associated with mid $\mathrm{SI}_{2}$ values around $10^{\circ}$ (Fig. 5a). Similarly, the $\mathrm{SI}_{2}$-RDI joint-PDF map indicates that the extreme total pressure radial distortion (RDI) events are not synchronous with the peak values of swirl distortion (Fig. 5b). The greatest SI values of approximately $22^{\circ}$ are associated with low intensity hub radial distortion $(\mathrm{RDI}<0)$ around $|\mathrm{RDI}|=0.008$ and 0.014 (Fig. $5 \mathrm{~b})$. In turn, the greatest $|\mathrm{RDI}|>0.02$ levels that occur at the hub are associated with low values of SI between $7^{\circ}$ and $13^{\circ}$. The peak tip radial distortion events are also uncorrelated with the extreme swirl distortion events (Fig. 5b). Therefore, peak values of swirl distortion are not synchronized with extreme events of either radial or circumferential total pressure distortion. This highlights the need to investigate both total pressure and swirl distortions in intake/engine compatibility assessments, as opposed to the extended practice of focusing just in total pressure distortion which has proven to result in compatibility problems when the swirl distortion is significant [10]. 


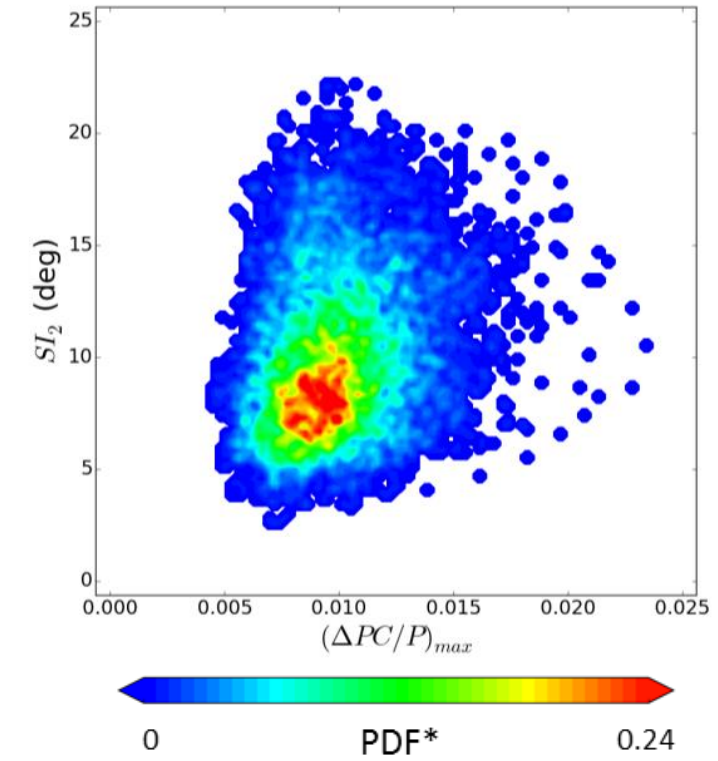

(a) $\mathrm{SI}_{2}-(\Delta \mathrm{PC} / \mathrm{P})_{\max }$

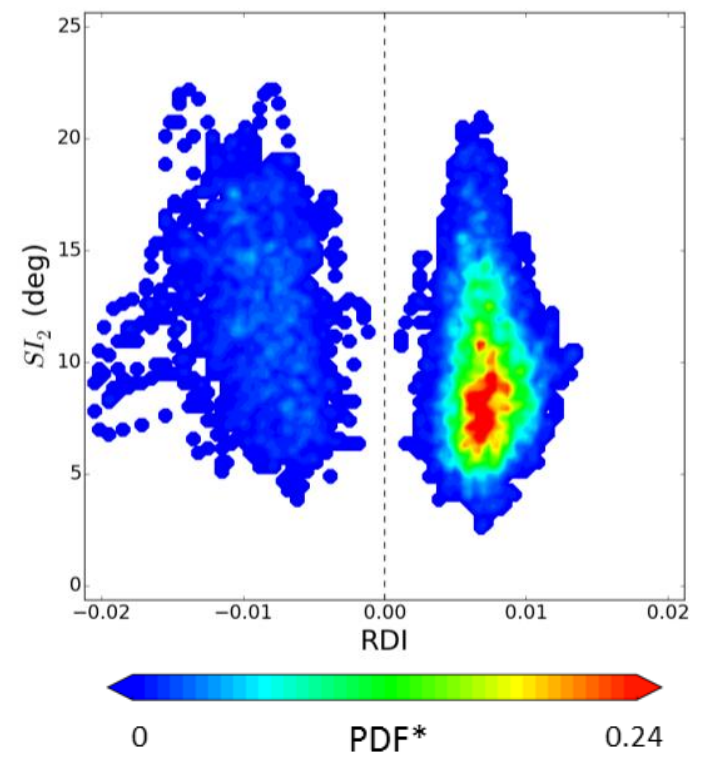

(b) $\mathrm{SI}_{2}-\mathrm{RDI}$

Fig. 5 Joint-PDF of swirl and total pressure distortion descriptors

\subsection{Distortion spectral analysis}

In addition to considering both the total pressure and swirl distortion, the fan response is also dependent upon the frequency associated with the flow distortion and only frequencies lower than a critical value could affect the fan stability [14]. However to the authors' knowledge there is no published information about the spectral characteristics of the swirl and total pressure distortion metrics. In this section, the spectrum of the circumferential $(\Delta \mathrm{PC} / \mathrm{P})_{\max }$ and radial RDI total pressure distortion descriptors, and swirl distortion intensity $\mathrm{SI}_{2}$ and directivity $\mathrm{SD}_{2}$ are assessed (Fig. 6). The Power Spectrum (PS) was computed with Welch's average periodogram method [35] with two non-overlapping segments, and the frequency resolution and maximum frequency in the PS were approximately $\Delta \mathrm{St}=0.01$ and $\mathrm{St}=36$, respectively. The PS was normalised by the variance of the corresponding time signal so that it represents the contribution of each individual frequency component to the overall variance. These contributions can be put in context with the standard-deviations of $\mathrm{SI}_{2}, \mathrm{SD}_{2},(\Delta \mathrm{PC} / \mathrm{P})_{\max }$ and $\mathrm{RDI}$ which are $3.2^{\circ}, 0.70,0.0023$ and 0.0020 , respectively. To put the distortion descriptors frequencies in context the well-known NASA Rotor 67 [36] is considered, and the critical non-dimensional frequency based on Cousin's guideline [15] is estimated to be approximately $\mathrm{St}=4.0$.

The swirl descriptors $\mathrm{SI}_{2}$ and $\mathrm{SD}_{2}$ show a single dominant peak at approximately $\mathrm{St}=1.06$ (Fig. 6a) and 0.53 (Fig. 6b), respectively. The radial $p_{0}$-distortion descriptor RDI shows two distinct frequencies at $\mathrm{St}=0.61$ and 1.09 , and the contributions from frequencies $\mathrm{St}>2.0$ are negligible (Fig. $6 \mathrm{c})$. The circumferential $p_{0^{-}}$-distortion descriptor $(\Delta \mathrm{PC} / \mathrm{P})_{\max }$ shows a much more broadband spectrum with contributions from higher frequencies, and two minor peaks can be identified at approximately $\mathrm{St}=0.67$ and 1.08 (Fig. 6d). Overall, the dominant frequencies for both total pressure and swirl distortion descriptors are below the estimated critical frequency of $\mathrm{St}=4.0$ (Fig. 6), and could have a destabilising effect in the fan.

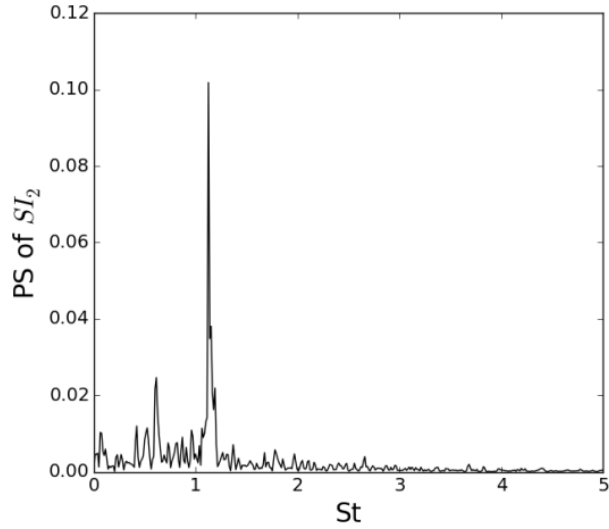

(a) PS of $\mathrm{SI}_{2}$

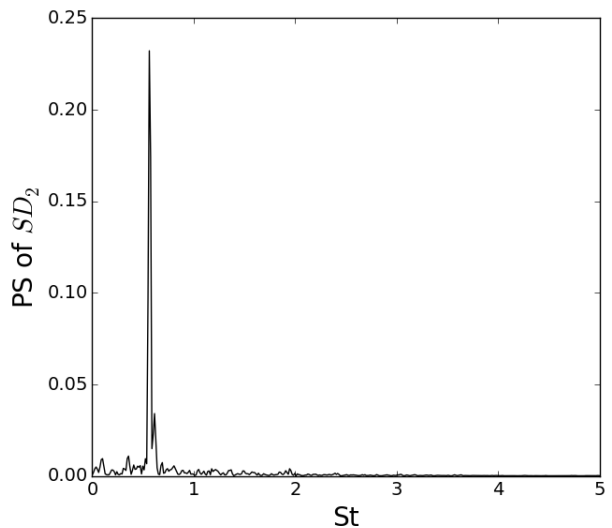

(b) PS of $\mathrm{SD}_{2}$ 


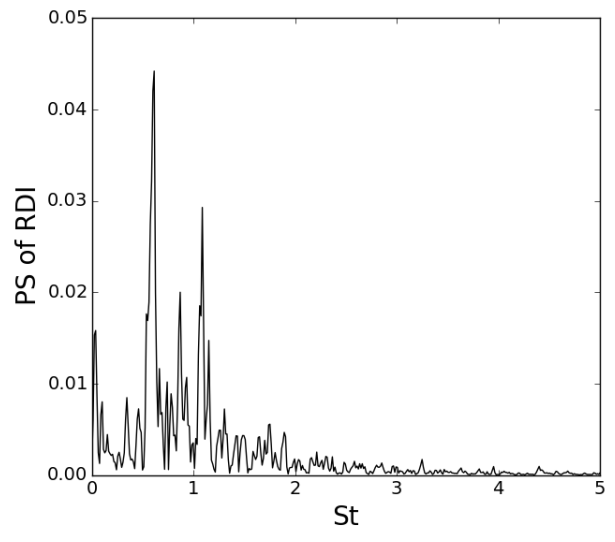

(c) PS of RDI

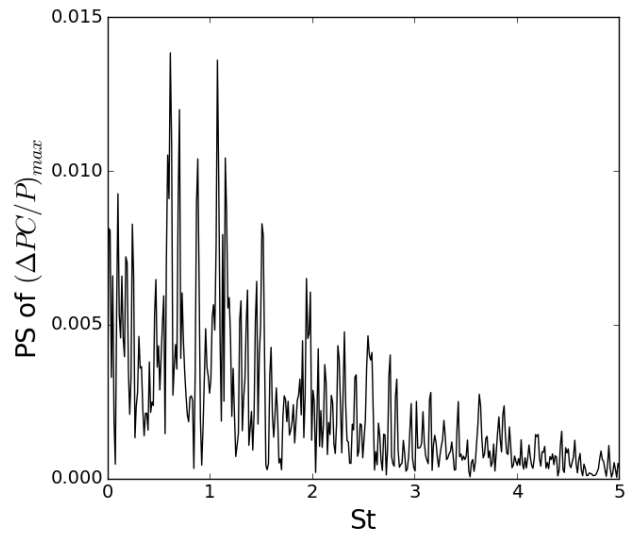

(d) $\mathrm{PS}$ of $(\Delta \mathrm{PC} / \mathrm{P})_{\max }$

Fig. 6 Power spectrum normalised by the variance of the overall time signal for the swirl and total pressure distortion descriptors

\subsection{Peak distortion duration}

The spectral analysis of the distortion metrics has indicated that the main frequencies are within the range in which they are expected to compromise the stability margin of the compression system. In the development programme of a propulsion system where the engine application is known, the intake data would normally be low-pass filtered to meet the response time scale of the compression system [34] and then the peak distortion value would be identified as the main concern for the engine stability [12]. However, in S-duct investigations the engine design is not always known a priori and the peak distortion levels are often reported without any low-pass filtering [19,20]. In this section it is proposed to report the peak distortion intensity as a function of its time scale or duration $t_{d}$. As suggested by Bowditch and Coltrin [11] a Moving Time-Averaging Window (MTAW) is applied to the unsteady signal of the considered distortion descriptor, and the corresponding peak value of the MTAW-averaged descriptor is then associated with a residence time $t_{d}$ equal to the size of the MTAW. The minimum MTAW size considered is equal to the original spacing between samples $\Delta t^{*}$, and the resulting signal is therefore identical to the original one. To put the results of this section in context, the minimum critical time of the open source Rotor 67 [36] has been estimated using Cousin's guideline [15] as approximately $t_{d} / \tau_{c}=0.25$, where $\tau_{c}=D_{A I P} /\langle\bar{w}\rangle_{A I P}$

This approach is applied to the $\mathrm{SI}_{2},(\Delta \mathrm{PC} / \mathrm{P})_{\max }$ and RDI descriptors, and the peak values associated with the different residence times $t_{d} / \tau_{c}$ are computed (Fig. 7). As expected, the peak levels for all the descriptors decrease monotonically as the characteristic duration time $t_{d} / \tau_{c}$ increases since the MTAW progressively filters out the high frequency spikes in the signals (Fig. 7). This permits the evaluation of the peak average distortion intensity to be expected to last a given residence time. For the original signal sampled at $\Delta t^{*}\left(t_{d} / \tau_{c}=0.01\right)$ the peak values of $\mathrm{SI}_{2},(\Delta \mathrm{PC} / \mathrm{P})_{\max }$ and $\mathrm{RDI}$ are $22.3^{\circ}, 0.0235$ and 0.0205 , respectively. However, these values are associated with a residence time below the estimated minimum critical value, and therefore are not expected to affect the fan stability. For the estimated minimum critical time $t_{d} / \tau_{c}=0.25$, the peak values of the descriptors are $19.5^{\circ}, 0.0166$ and 0.0172 for $\mathrm{SI}_{2}$, $(\triangle \mathrm{PC} / \mathrm{P})_{\max }$ and RDI, respectively (Fig. 7). Therefore, an inlet/engine compatibility assessment based on the peak values as obtained from the raw unsteady signal would over-estimate the peak distortion levels by approximately $14 \%, 42 \%$ and $19 \%$ for $\mathrm{SI}_{2},(\Delta \mathrm{PC} / \mathrm{P})_{\max }$ and RDI, respectively, and could therefore result in an over-conservative design. These figures have been obtained as the difference between the peak levels associated with the raw data and the critical time, divided by the value associated with the critical time. Therefore, the proposed approach offers a more general way to report peak distortion data from intake investigations when the details of the engine design are not known a priori. 


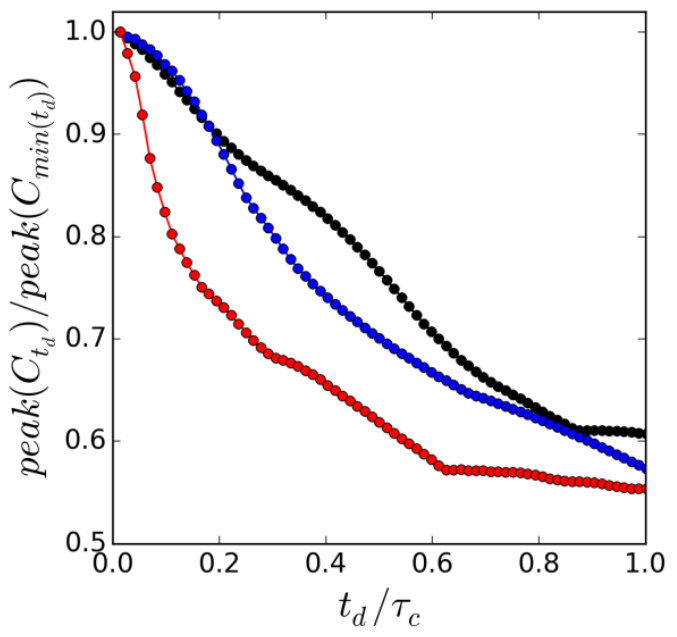

Fig. 7 Peak value of the distortion metric $\mathrm{C}$ associated with different durations $t_{d}$, normalized by the level corresponding to the raw signal for which $t_{d}=\min \left(t_{d}\right)$. Black: $\mathrm{C}=\mathrm{SI}_{2}$, blue: $\mathrm{C}=\mathrm{RDI}$, red: $\mathrm{C}=(\Delta \mathrm{PC} / \mathrm{P})_{\max }$

The time-duration analysis is also undertaken for $\mathrm{SD}_{2}$ (Fig. 8). The duration of the extreme bulk $\left(\left|\mathrm{SD}_{2}\right|=1\right)$ and twin $\operatorname{swirl}\left(\left|\mathrm{SD}_{2}\right|=0\right)$ patterns has been assessed based on the time-signal of the absolute-valued $\mathrm{SD}_{2}$. The MTAW approach described earlier in this subsection has been applied and the maximum peak and minimum value of the associated time-signal have been calculated and related to the corresponding time duration $t_{d}$ (Fig. 8). Nominal bulk swirl events $\left(\left|\mathrm{SD}_{2}\right|=1\right)$ observed in the raw signal are associated with timescales as large as $t_{d} / \tau_{c}=0.43$ (Fig. 8) and therefore could affect the engine stability for the estimated minimum critical time of $t_{d} / \tau_{c}=0.25$. Nominal twin swirl events $\left(\left|\mathrm{SD}_{2}\right|=0\right)$ are also observed in the raw data and only minor deviations occur when the estimated critical time scale of $t_{d} / \tau_{c}=0.25$ is considered, for which the minimum value of $\left|\mathrm{SD}_{2}\right|$ slightly increases up to 0.05 . Therefore both bulk and twin swirl patterns are associated with time scales sufficiently large to affect the stability of the compression system.

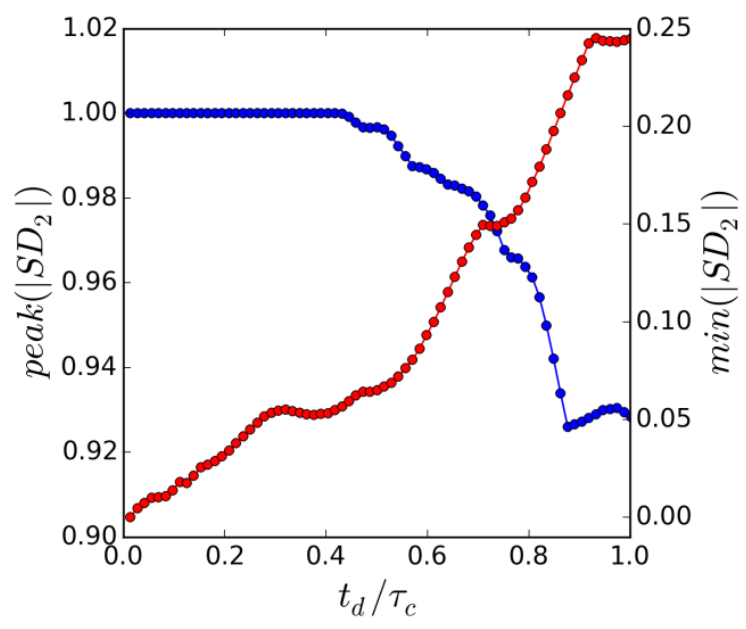

Fig. 8 Time duration $t_{d}$ associated with bulk and twin swirl patterns represented by the peak (blue) and minimum (red) absolute values of the $\mathrm{SD}_{2}$

\subsection{Extreme Value Theory}

The peak value of the temporal distribution of a given distortion descriptor has become a parameter of major importance for inlet/engine compatibility assessments. However the peak distortion depends on the acquisition time and is therefore an inconsistent estimator [12]. A typical acquisition time in flow distortion experiments range from seconds to a few minutes. Even if an acceptable peak distortion value has been observed during this period of time, a greater value at later instants of time could trigger engine instabilities. Based on a finite sample of observations Extreme Value Theory (EVT) (Section 2.5) permits the estimation of the probability of extreme distortion events which exceed the maximum levels recorded in the measured dataset [24]. In this section the EVT is applied to the swirl distortion descriptor SI (Eq. 1) distortion for the first time. A fundamental assumption of the EVT is that the samples are statistically independent [24] and therefore the EVT analysis can be performed on the SPIV data which is acquired at a sampling frequency of $3 \mathrm{~Hz}\left(\mathrm{St}=7.5 \times 10^{-3}\right)$, much lower than the fundamental frequencies of the flow (Fig. 6). DDES computational data is not used in this section since adjacent samples are obviously time dependent. The EVT is also applied to the proposed VDC60 (Eq. 4) distortion descriptor to have an estimation of the peak levels of velocity distortion. 


\subsubsection{Swirl Distortion Intensity}

To apply EVT to the Swirl Intensity $\mathrm{SI}_{2}$, whose probability distribution shows a peak value of $18.4^{\circ}$ (Fig. $9 \mathrm{a}$ ), a threshold $U$ has to be selected above which the observations are considered as the exceedances to be fitted by the model (Section 2.5). Several criteria have been proposed to select an optimum threshold. In particular, above a valid threshold the mean excess should be linear with threshold variations, and the model parameters $\left\{\xi, \sigma^{*}=\sigma-\xi U\right\}$ should be constant after allowance for confidence intervals [24]. These conditions are checked in this investigation for the selected threshold. However these conditions are difficult to be expressed as a quantitative expression and are often accomplished by a wide range of thresholds [24]. In this work the threshold of $8.0^{\circ}$ is chosen to minimise the root-mean-square (RMS) error between the observed and predicted quantiles (Fig. 9b), which is the most stringent criterion for model diagnosis [24]. The good agreement of the EVT model with the measured data is checked by comparing the probability distribution (Fig. 10a) and quantiles (Fig. 10b) of the measured and modelled exceedances.

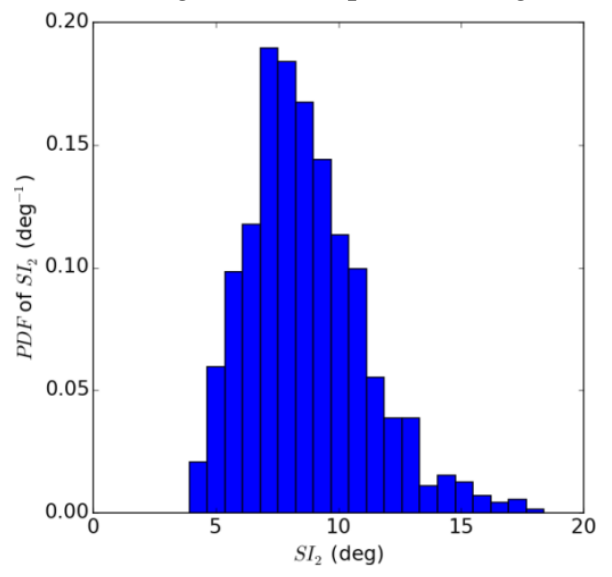

(a) PDF

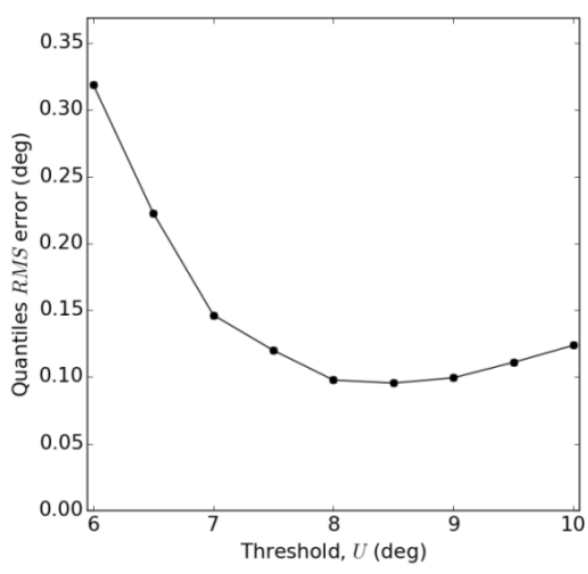

(b) Quantiles error

Fig. 9 PDF and threshold selection for the application of EVT on $\mathrm{SI}_{2}$

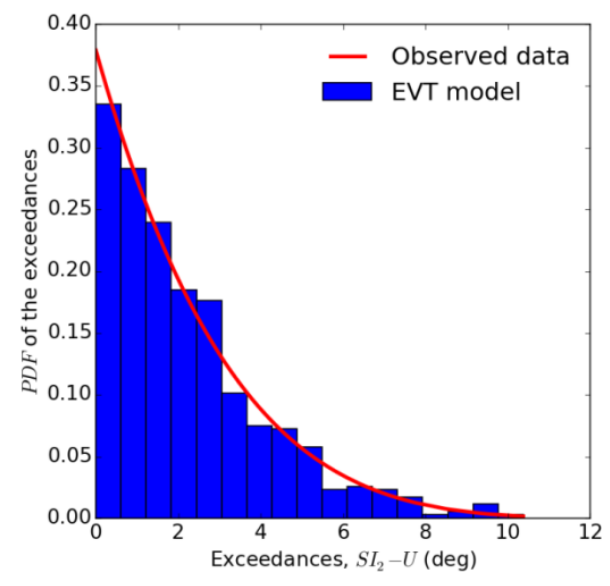

(a) PDF

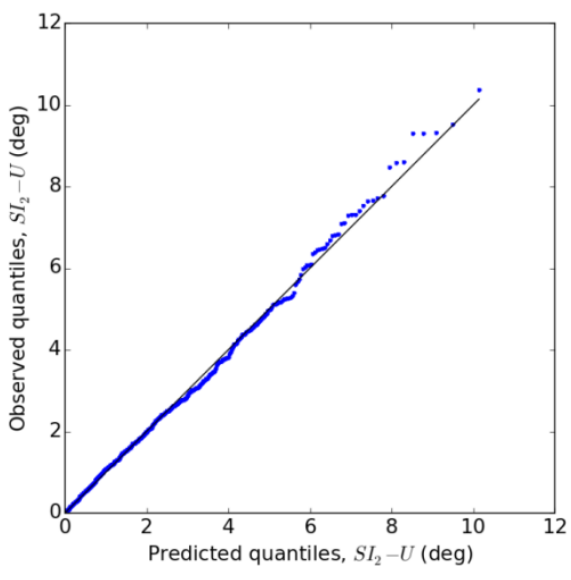

(b) Quantiles

Fig. 10 Comparison between observed data and the EVT model predictions for $\mathrm{SI}_{2}$

The model prediction is usually reported as a return plot which shows the different peak $\mathrm{SI}_{2}$ values associated with different probability levels $p$, usually expressed as the reciprocal $m=1 / p$ (Fig. 11a). The parameter $m$ represents the number of observations for which the corresponding extreme level is expected to be exceeded once on average. The 95\% confidence intervals and the empirical data are also illustrated for comparison with the model predictions (Fig. 11a). As expected, the confidence intervals increase as the probability $p$ of the extreme event reduces, i.e. as $m$ increases. This is due to the greater uncertainty associated with the prediction of less probable extreme events, and it is usually recommended not to attempt extrapolation beyond 100 times the basic observational time [12]. In this case this equates to $\mathrm{m}=10^{5}$, and the expected peak event is approximately $\mathrm{SI}_{2}=20.9^{\circ} \pm 2.4^{\circ}$ (Fig. $11 \mathrm{a}$ ) which is expected to occur once every $10^{5}$ observations. Another parameter of interest is the upper bound predicted by the model of $23.2^{\circ}$ (Fig. 11a). This value has to be taken with caution since the confidence intervals can become unacceptably large for very rare events. However it has been used in previous investigations as a more consistent indicator than the observed peak value which highly depends on the acquisition time and sampling frequency [12]. The EVT has also been applied to the SI considered at different radial positions (Fig. 11b) and in each case the threshold has been selected to minimize the quantiles RMS. For a similar number of approximately 500 exceedances, the uncertainty in the peak $S I_{i}$ values predictions at a given level of probability $m$ increases for the inner radial positions in the AIP (Fig. 11b). This indicates that for a given uncertainty requirement, more samples have to be acquired in order to predict peak values of swirl distortion in the hub than in the tip region. 


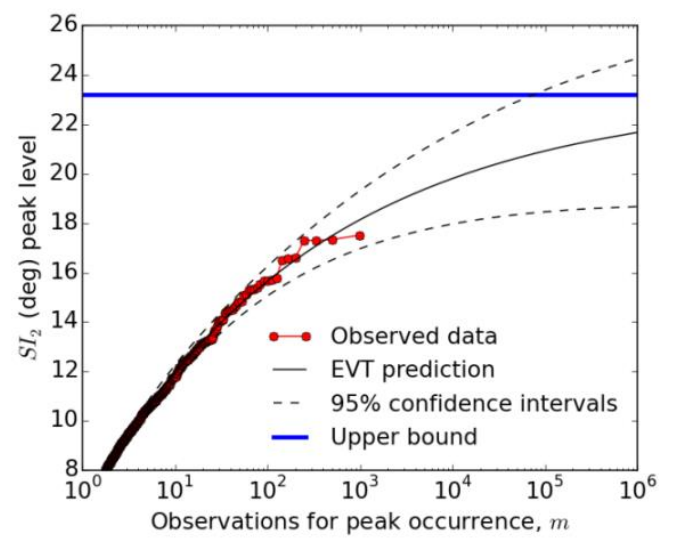

(a) $\mathrm{SI}_{2}$

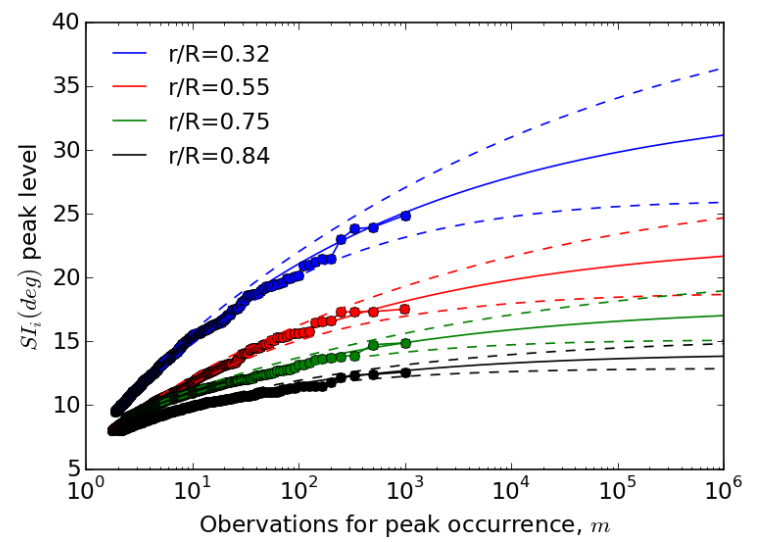

(b) $\mathrm{SI}_{\mathrm{i}}$ at different radial positions

Fig. 11 Predicted SI extreme values for different probability levels

The statistical convergence of the EVT method has been assessed based on the shape $\xi$ (Fig. 12a), scale $\sigma$ (Fig. 12b) and upper bound (Fig. 12c) of the $\mathrm{SI}_{2}$ model (Section 2.5). These parameters have been calculated for different number of snapshots with a constant threshold of $8.0^{\circ}$ (Fig. 9b). For more than 500 snapshots the model parameters $\xi$ and $\sigma$ do not change significantly, and the upper bound predicted by the model remains within $3 \%$ of the value obtained with 1000 snapshots (Fig. 12). Similar results are obtained for the others distortion metrics considered. Therefore, a minimum of 500 snapshots is recommended to apply EVT on these distortion descriptors.

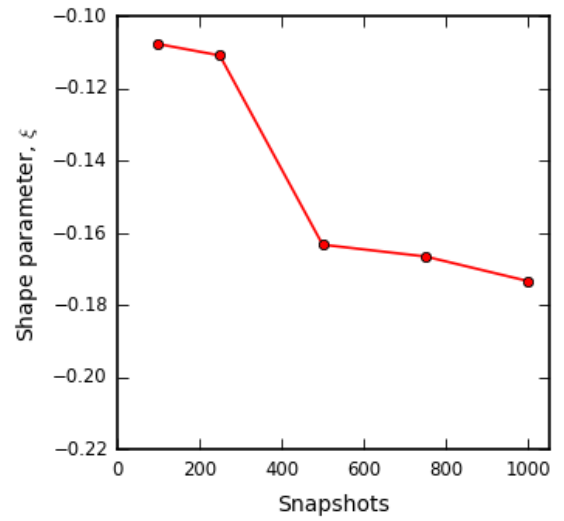

(a) Shape parameter

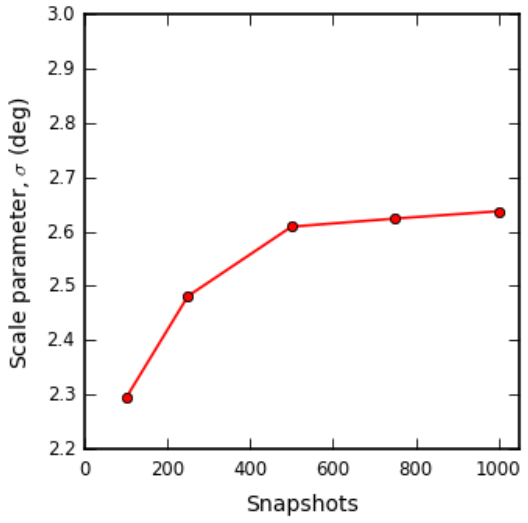

(b) Scale parameter

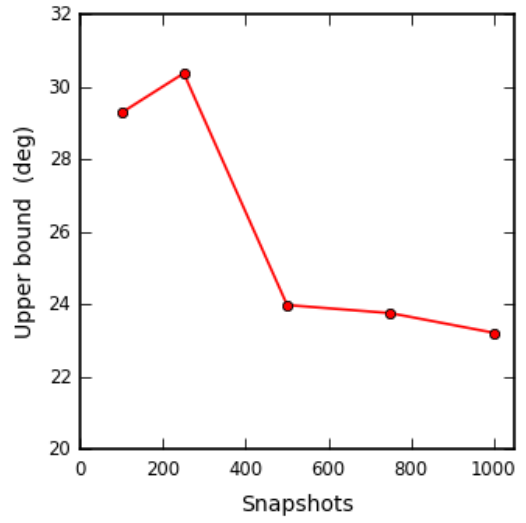

(c) Upper bound

Fig. 12 Statistical convergence of the EVT model parameters for $\mathrm{SI}_{2}$

\subsubsection{Velocity distortion descriptor VDC60}

The VDC60 velocity distortion metric (Eq. 4) has been proposed for analyses were the velocity field is measured and total pressure data are not available, such as for high resolution SPIV experiments [22,23]. However, it is important to understand the relationship between the unsteady VDC60 and the more established DC60 (Eq. 3). The DDES solution contains both total pressure and velocity data and therefore allows for such assessment. The time-signal of VDC60 follows relatively closely that of DC60 (Fig. 13), and these two metrics show a significant cross-correlation coefficient of 0.88 . The mean, standard deviation and maximum values of these two metrics are also in good agreement, with $0.2556,0.0895$ and 0.6709 for DC60, and 0.2607, 0.0895 and 0.6114 for VDC60, respectively. In this context, it is proposed to consider VDC60 as a useful distortion metric for SPIV-type velocity datasets.

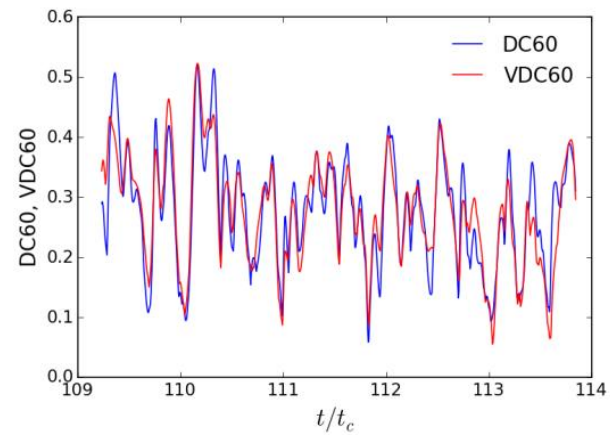


Fig. 13 Comparison between DC60 and the velocity-based metric VDC60

The EVT is applied to the velocity-based VDC60 (Eq. 4) obtained from the SPIV data, which shows a peak value of 0.59 (Fig. 14a). The threshold is selected at 0.32 and the EVT model shows a good agreement with the observed data (Fig. 14b). The predicted level at $\mathrm{m}=10^{5}$ is of approximately VDC60 $=0.61 \pm 0.03$ (Fig. 14b), and the upper bound of the distribution is 0.62 which is about $6 \%$ greater than the observed peak value. Overall, EVT permits a more consistent inlet/engine compatibility assessment, where the design can be based on a given extreme level to be expected with a prescribed probability $p$ within a certain confidence interval. The compatibility assessment can then account for rare extreme events which have not been observed in the empirical data due to the low associated probabilities of occurrence. This approach has the potential to de-risk the inlet/engine development and to reduce the time involved in time consuming and expensive experimental time usually performed during the inlet/engine compatibility assessment. In addition, it removes the subjectivity to the frequency and time of acquisition.

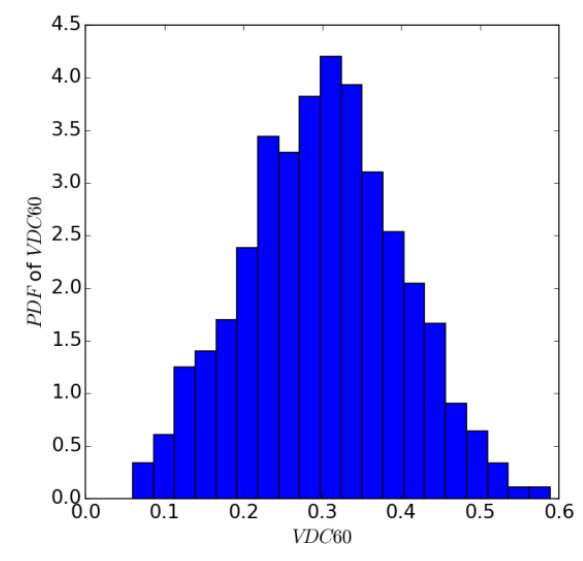

(a) PDF

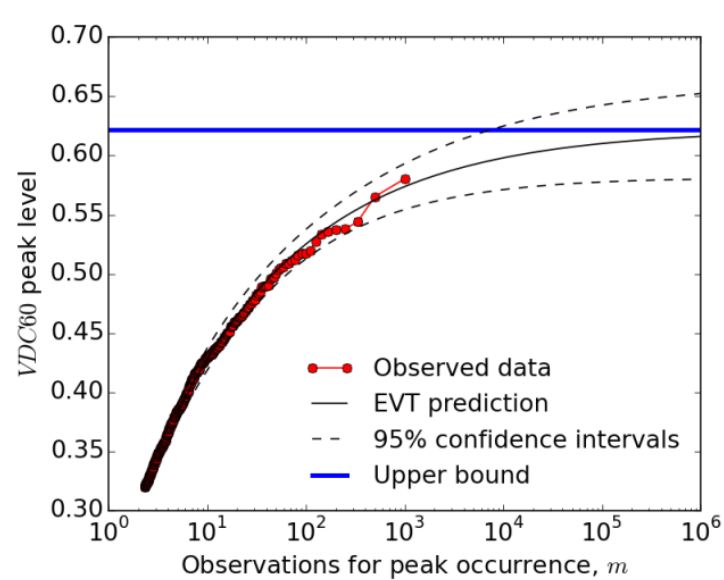

(b) Return plot

Fig. 14 PDF and predicted extreme values for VDC60

\section{Conclusions}

The data from a previous Delayed Detached-Eddy Simulation and Stereo Particle Image Velocimetry investigation of the flow field in an S-duct intake at $\mathrm{M}_{\mathrm{ref}}=0.27$ have been used to propose a range of analytical methods for the study of unsteady flow distortion in complex intakes. Time-resolved DDES data has been used to provide insight about peak distortion duration, distortion spectral characteristics, and the relation between swirl and total pressure distortions, which is otherwise not possible with the low-bandwidth SPIV experimental data.

The typical unsteady swirl distortion patterns at the outlet of the S-duct intake have been identified with a conditional averaging based on the Swirl Directivity metric. For both DDES and SPIV data the most intense swirl distortion events were associated with a single dominant rotating structure at the AIP. The detailed knowledge of these unsteady patterns is of importance since they are typically replicated with steady distortion generators to test the engine tolerance to distortion. The frequency of the distortion has a critical effect on the fan response, and in this work the spectral characteristics of the distortion descriptors are assessed for the first time. The swirl distortion descriptors show a single dominant frequency at approximately St=1.06 and 0.53 for the Swirl Intensity and Swirl Directivity, respectively. The radial and particularly circumferential total pressure distortion descriptors show a more broadband spectrum. Overall, the main frequencies for all the distortion descriptors are below the estimated maximum critical frequency of a typical fan $\mathrm{St}=4.0$, and therefore could affect the fan operability. A more general way to report peak distortion data from intake investigations when the details of the engine design are not known a priori has been proposed. In these cases the response time of the compression system is not known and often the peak distortion data is calculated from the raw signals without taking into account the duration of these events. Therefore it is considered pertinent to report the peak distortion levels as a function of the associated time scale, so that the results can be interpreted for a range of engine applications with different response times. For the investigated case, for example, the peak values of SI, $(\triangle \mathrm{PC} / \mathrm{P})_{\max }$ and RDI obtained from the raw signal were over-predicted by approximately $14 \%$, $42 \%$ and $19 \%$ compared with the peak values averaged over a typical minimum critical fan time response. This highlights the importance of considering the time duration in the analysis of the peak distortion levels. The DDES data was also used to assess for the first time the relation between unsteady swirl and both radial and circumferential total pressure distortion, and it was demonstrated that peak swirl distortion events are not synchronous with either circumferential or radial total pressure peak distortions. This highlights the need to assess together total pressure and swirl distortion.

The Extreme Value Theory was used for the prediction of peak distortion values beyond the maximum levels observed in the acquired dataset. The measured dataset of 1000 snapshots could only regularly capture distortion peaks that occur with a probability of $\mathrm{p}=10^{-3}$. Based on typical extrapolation guidelines, EVT permits a prediction of the peak distortion levels associated with a probability as low as $\mathrm{p}=10^{-5}$. The experimental observation of peaks associated with $\mathrm{p}=10^{-5}$ would require the acquisition of $10^{5}$ snapshots and about two orders of magnitude longer testing times, with the associated cost penalty. For example, the predicted peak value of swirl 
intensity to be found in a dataset of $10^{5}$ samples was $\mathrm{SI}_{2}=20.9^{\circ} \pm 2.4^{\circ}$, which is about $14 \%$ greater than the peak value of $18.4^{\circ}$ in the measured dataset of $10^{3}$ samples. The VDC60 parameter has been proposed as a pertinent distortion metric when the velocity field is measured and total pressure data is not available, such as in SPIV experiments. The EVT was also applied to this descriptor, and the predicted peak value at $\mathrm{p}=10^{-5}$ was VDC60 $=0.61 \pm 0.03$, which is within $4 \%$ of the observed peak of 0.59 .

Overall, this work proposes a range of analytical techniques applicable to unsteady distortion investigations. A way to quantify the peak distortion as a function of the associated time scale has been proposed which is expected to be useful in those intake investigations where the engine is not known a priori. The duration of the peak levels of unsteady swirl distortion has been assessed for the first time. The main frequencies of the total pressure and swirl distortion descriptors have been identified. Extreme Value Theory has been applied to predict extreme distortion events whose observation would otherwise require prohibitive testing times. The relation between peak total pressure and swirl distortion events has been assessed, and the flow patterns that promote the most intense swirl distortion events have been identified.

\section{References}

[1] Kawai, R. T., Friedman, D. M., and Serrano, L., "Blended Wing Body (BWB) Boundary Layer Ingestion (BLI) Inlet Configuration and System Studies," NASA Technical Report CR-2006-214534, Dec. 2006.

[2] Kim, H., and Liou, M-S., "Shape design optimization of embedded engine inlets for N2B hybrid wing-body configuration," Aerospace Science and Technology, Vol. 30, No. 1, Oct. 2013, pp. 128-149. Doi: 10.1016/j.ast.2013.07.011

[3] Stenning, A. H., "Rotating Stall and Surge," Journal of Fluids Engineering, Vol. 102, No. 1, Mar. 1980, pp. 14-20. Doi:10.1115/1.3240618

[4] Stevens, C. H., Spong, E. D., and Hammock, M. S., "F-15 Inlet/Engine Test Techniques and Distortion Methodologies Studies. Volume I Technical Discussion,” NASA Contractor Report 144866, Jun. 1978

[5] Paul, A. R., Ranjan, P., Patel, V. K., and Jain, A., "Comparative studies on flow control in rectangular S-duct diffuser using submerged-vortex generators," Aerospace Science and Technology, Vol. 28, No. 1, Jul. 2013, pp. 332-343. Doi: 10.1016/j.ast.2012.11.014

[6] Harouni, A. G., "Flow control of a boundary layer ingesting serpentine diffuser via blowing and suction," Aerospace Science and Technology, Vol. 39, Dec. 2014, pp. 472-480. Doi: 10.1016/j.ast.2014.05.016

[7] Keerthi, M. C., and Kushari, A., "Effectiveness of vortex generator jets and wall suction on separated flows in serpentine-duct diffuser," Aerospace Science and Technology, Vol. 34, Apr. 2014, pp. 12-19. Doi: 10.1016/j.ast.2014.01.013

[8] Gan, W., and Zhang, X., "Design optimization of a three-dimensional diffusing S-duct using a modified SST turbulence model," Aerospace Science and Technology, Vol. 63, Apr. 2017, pp. 63-72. Doi: 10.1016/j.ast.2016.12.016

[9] Society of Automotive Engineers, "Inlet Total-Pressure-Distortion Considerations for Gas-Turbine Engines," SAE Aerospace Information Report 1419a, Warrendale, PA, USA, 1999.

[10] Society of Automotive Engineers, "A Methodology for Assessing Inlet Swirl Distortion," SAE Aerospace Information Report 5686, Warrendale, PA, USA, 2007.

[11] Bowditch, D. N., and Coltrin, R. E., “A Survey of Inlet/Engine Distortion Compatibility,” NASA TM-83421, June 1983.

[12] Jacocks, J. L., and Kneile, K. R., "Statistical Prediction of Maximum Time-Variant Inlet Distortion Levels," Arnold Engineering Development Center, Technical Report AEDC-TR-74-121, Tennessee, USA, Jan. 1975.

[13] Biesiadny, T. J., Braithwaite, W. M., Soeder, R. H., and Abdelwahab, M., "Summary of Investigations of Engine Response to Distorted Inlet Conditions," NASA TM-87317, Sep. 1986.

[14] Breuer, T., and Bissinger, N. C., "Basic Principles: Gas Turbine Compatibility - Gas Turbine Aspects," Encyclopedia of Aerospace Engineering, Dec. 2010. Doi: 10.1002/9780470686652.eae573

[15] Cousins, W., T., "History, Philosophy, Physics and Future Directions of Aircraft Propulsion System / Inlet Integration," Proceedings of ASME Turbo Expo 2004: Power for Land, Sea, and Air, Vol. 2, GT2004-54210, American Society of Mechanical Engineers, Vienna, Austria, June 2004, pp. 305-320. Doi: 10.1115/GT2004-54210

[16] Hercock, R. G., and Williams, D. D., "Distortion-Induced Engine Stability: Aerodynamic Response," AGARD Lecture Series No. 72 , Paper No. 3, Oct. 1974

[17] Williams, D. D., "Review of current knowledge on engine response to distorted inflow conditions," AGARD-CP-400 Engine Response to Distorted Inflow Conditions, Paper No. 1, Mar. 1987

[18] MacMiller, C. J., and Haagenson, W. R., "Unsteady inlet distortion characteristics with the B-1B," AGARD-CP-400 Engine Response to Distorted Inflow Conditions, Paper No. 16, Mar. 1987

[19] Garnier, E., "Flow Control by Pulsed Jet in a Curved S-Duct: A Spectral Analysis," AIAA Journal, Vol. 53, No. 10, Oct. 2015 , pp. $2813-2827$. Doi: $10.2514 / 1 . J 053422$

[20] MacManus, D. G., Chiereghin, N., Gil-Prieto, D., and Zachos, P., "Complex Aeroengine Intake Ducts and Dynamic Distortion,” AIAA Journal, Vol. 55, No. 7, Jul. 2017, pp. 2395-2409. Doi: 10.2514/1.J054905

[21] Wellborn, S. R., Reichert, B. A., and Okiishi, T. H., "Study of the Compressible Flow in a Diffusing S-Duct," AIAA Journal of Propulsion and Power, Vol. 10, No. 5, Sept.-Oct. 1994, pp. 668-675. Doi: 10.2514/3.23778

[22] Zachos, P. K., MacManus, D. G., Gil-Prieto, D., and Chiereghin, N., "Flow Distortion Measurements in Convoluted Aeroengine Intakes," AIAA Journal, Vol. 54, No. 9, Sep. 2016, pp. 2819-2832. Doi: 10.2514/1.J054904

[23] Gil-Prieto, D., MacManus, D. G., Zachos, P. K., Tanguy, G., and Menzies, K. R., "Convoluted Intake Distortion Measurements Using Stereo Particle Image Velocimetry,” AIAA Journal, Vol. 55, No. 6, Apr. 2017, pp. 1878-1892. Doi: 10.2514/1.J055467

[24] Coles, S., An Introduction to Statistical Modeling of Extreme Values, Springer Series in Statistics, Springer-Verlag London, 2001, Chap. 4, pp. 74-91. Doi: 10.1007/978-1-4471-3675-0

[25] Wieneke, B., "Stereo-PIV using self-calibration on particle images," Experiments in Fluids, Vol. 39, No. 2, May 2005, pp. 267-280. Doi:10.1007/s00348-005-0962-z

[26] Raffel, M., Willert, C., Wereley, S., and Kompenhans, J., Particle Image Velocimetry: A Practical Guide, $2^{\text {nd }}$ ed., Springer-Verlag, Berlin, 2007, Chap. 5, pp. 164-176. Doi: 10.1007/978-3-540-72308-0 
[27] Motycka, D. L., "Reynolds Number and Fan/Inlet Coupling Effects on Subsonic Transport Inlet Distortion," Journal of Propulsion and Power, Vol. 1, No. 3, 1985, pp. 229-234. Doi:10.2514/3.22785

[28] Hodder, B. K., “An Investigation of Engine Influence on Inlet Performance,” NASA CR-166136, 1981

[29] Gil-Prieto, D., MacManus, D. G., Zachos, P. K., Tanguy, G., Wilson, F., and Chiereghin, N., "Delayed Detached-Eddy Simulation and Particle Image Velocimetry Investigation of S-Duct Flow Distortion,” AIAA Journal, Vol. 55, No. 6, Mar. 2017, pp.1893-1908. Doi: 10.2514/1.J055468

[30] Spalart, P. R., Deck, S., Shur, M. L., Squires, K. D., Strelets, M. K., and Travin, A., "A New Version of Detached-Eddy Simulation, Resistant to Ambiguous Grid Densities," Theoretical and Computational Fluid Dynamics, Vol. 20, No. 3, May 2006, pp. 181-195. Doi:10.1007/s00162006-0015-0

[31] Seddon, J., and Goldsmith, E. L., Intake Aerodynamics, 2nd ed., Blackwell Science, Boston, 1999, Chap. 11, pp. $281-284$.

[32] Castillo, E., Hadi, A. S., Balakrishnan, N., and Sarabia, J. M., Extreme Value and Related Models with Applications in Engineering and Science, Wiley Series in Probability and Statistics, John Wiley \& Sons, Hoboken, New Jersey, USA, 2005, Chap. 10, p. 269.

[33] Williams, D. D., and Yost, J. O., "Some aspects of inlet/engine flow compatibility," Aeronautical Journal, Vol. 77, No. 753, Sep. 1973, pp. 483492. Doi: $10.1017 / \mathrm{S} 0001924000041610$

[34] Kidman, D. S., Reagan, P. V., and Malloy, D. J., "Comparison of Inlet Compatibility Results From Subscale Wind Tunnel and Full-Scale Flight Tests of the F/A-22 Aircraft with the F119-PW-100 Engine," Proceedings of the 17th Symposium on Air Breathing Engines, ISABE-2005-1169, International Society of Air Breathing Engines, Munich, Germany, 2005.

[35] Welch, P. D., "The Use of Fast Fourier Transform for the Estimation of Power Spectra: A Method Based on Time Averaging Over Short, Modified Periodograms," IEEE Transactions on Audio and Electroacoustics, Vol. 15, No. 2, Jun. 1967, pp. 70-73. Doi: 10.1109/TAU.1967.1161901

[36] Strazisar, A. J., Wood, J. R., Hathaway, M. D., and Suder, K. L., "Laser Anemometer Measurements in a Transonic Axial-Flow Fan Rotor," NASA Technical Paper 2879, Nov. 1989. 
2017-10-27

\title{
Assessment methods for unsteady flow distortion in aero-engine intakes
}

\author{
Gil-Prieto, Daniel
}

Elsevier

Gil-Prieto D, MacManus DG, Zachos PK, Bautista A, Assessment methods for unsteady flow distortion in aero-engine intakes, Aerospace Science and Technology, Volume 72, January 2018, Pages 292-304

http://dx.doi.org/10.1016/j.ast.2017.10.029

Downloaded from Cranfield Library Services E-Repository 